Pacific

Journal of

Mathematics

CR APPROXIMATION ON A NONRIGID HYPERSURFACE GRAPH IN $\mathrm{C}^{n}$

Al Boggess and Roman Dwilewicz 


\title{
CR APPROXIMATION ON A NONRIGID HYPERSURFACE GRAPH IN $\mathrm{C}^{n}$
}

\author{
Al Boggess and Roman Dwilewicz
}

Let $M$ be a hypersurface in $\mathrm{C}^{n}$ that is the graph over a $(2 n-1)$-linear real space. The main result of the paper is that any $\mathrm{CR}$ function on $M$ can be uniformly approximated on compact subsets by entire functions on $\mathrm{C}^{n}$.

\section{Introduction}

In $[\mathbf{B T}]$, Baouendi and Treves prove a local CR approximation result that says (in particular) that $\mathrm{CR}$ functions on a $\mathrm{CR}$ submanifold can be locally approximated by entire functions. The global version of this theorem is false, as seen by the example $M=\left\{(z, w) \in \mathbf{C}^{2} ;|z|=1\right\}$ - the function $f(z, w)=1 / z$ is clearly CR on $M$ but cannot be approximated uniformly on compact subsets of $M$ by entire functions. A natural question is whether a global version of the CR approximation theorem holds in cases where there are no topological obstructions. In this work, we establish a partial answer to this question by showing that the global CR approximation theorem holds on any smooth real hypersurface that is globally presented as a graph.

To precisely state our theorem, let $\mathbf{C}^{n}$ be given coordinates $(z, w) \in$ $\mathbf{C} \times \mathbf{C}^{n-1}$. Let $h: \mathbf{R} \times \mathbf{R}^{n-1} \times \mathbf{R}^{n-1} \rightarrow \mathbf{R}$ be a smooth real-valued function and let $M$ be its graph:

$$
M=\left\{(z, w)=(h(y, u, v)+i y, u+i v) \in \mathbf{C} \times \mathbf{C}^{n-1}\right\} .
$$

Our goal is to prove the following theorem:

Theorem 1. Given a compact set $K \subset \mathbf{C}^{n}$, there is a compact set $K^{\prime} \subset \mathbf{C}^{n}$ (with $K \subset K^{\prime}$ ), such that if $f$ is continuous and $\mathrm{CR}$ on a neighborhood of $M \cap K^{\prime}$, then there is a sequence of entire functions on $\mathbf{C}^{n}$ that converges to $f$ uniformly on $M \cap K$.

Earlier work (see $[\mathbf{B}]$ ) established this theorem in the case where the graphing function $h$ is rigid (i.e., independent of $y$ ) and has polynomial growth. Earlier results on global CR approximation, see for example [DG], required additional technical assumptions (including certain convexity restrictions). Global approximation on totally real submanifolds of smooth functions by holomorphic functions has been considered by many authors, including $[\mathbf{H W}]$ and $[\mathbf{C h}]$. 
This work only handles the hypersurface case. The analogous theorem for higher codimension is still open and it does not appear that the techniques in this work can be easily modified to handle the case of higher codimension.

\section{Outline of proof}

As with the local version of the CR approximation theorem, the idea is to first integrate the given $\mathrm{CR}$ function $f(z, w)$ against a kernel that approximates the identity. The kernel will be entire in all variables, but is supported along a slice of $M$ that moves as the point $(z, w)$ moves (in a sort of Radon transform style). Since the slices don't depend holomorphically on $(z, w)$, the resulting approximating sequence is not a priori holomorphic. Thus, the next step is to show (with the help of Stokes' Theorem) that the slice can be fixed, independently of the point $(z, w)$. All of this analysis is done locally at first and then pieced together globally with a correction term that involves solving a $\bar{\partial}$ problem with estimates (in a Cousin-type fashion).

\section{Definition of kernel}

In $[\mathbf{B T}]$, an approximation to the identity kernel is used that somewhat looks like a heat kernel in $\mathbf{C}^{n}$. Our kernel also looks like a heat kernel but with the new feature of involving an extra complex parameter that gets integrated along an infinite ray in $\mathbf{C}$ (somewhat like a complex version of the Laplace transform). This extra parameter will allow us to handle global approximation. More specifically, for a continuous function $f$ on $M$, we consider the sequence

$$
E_{\epsilon}(f)(z, w)=\frac{C}{\epsilon^{n}} \int_{(\zeta, \eta) \in M_{u}} \int_{\alpha \in C_{\theta}} f(\zeta, \eta) E_{\epsilon}(\zeta, \eta, \alpha, z, w) \alpha^{n} d \alpha d \zeta d \eta
$$

where the kernel $E_{\epsilon}$ is given by

$$
E_{\epsilon}(\zeta, \eta, \alpha, z, w)=\exp \left(\alpha^{2}\left(\frac{\zeta-z}{\epsilon}\right)^{2}+\alpha^{2} \Lambda\left(\frac{w-\eta}{\epsilon}\right)^{2}-\alpha^{p}\right) .
$$

Here, $\alpha, \zeta \in \mathbf{C}$ and $\eta \in \mathbf{C}^{n-1}$ will be the variables of integration; $(z, w)$ is a point in $\mathbf{C} \times \mathbf{C}^{n-1}=\mathbf{C}^{n} ; \epsilon$ is a real parameter that will later converge to zero; and $\Lambda$ is a fixed positive real number (chosen below). The term $(w-\eta)^{2}$ stands for the sum of the squares of the components of $w-\eta \in \mathbf{C}^{n-1}$ (without any conjugates). The power $p$ will be a real number slightly larger than 2 and will be chosen below. The domain of integration involves $M_{u}$, which is the intersection of $M$ with the slice $\left\{(\zeta, \eta) \in \mathbf{C} \times \mathbf{C}^{n-1}\right.$; Re $\left.\eta=u\right\}$ where $u$ is the real part of $w$. The other component of the domain of integration is the ray $C_{\theta}=\left\{\alpha=r e^{i \theta} ; r>0\right\}$, where the angle $\theta$ will be between $-\frac{\pi}{4}$ and $\frac{\pi}{4}$ and its choice below will be determined by the desire to make the real part of the exponent of the kernel as negative as possible (so that the 
resulting integral will converge). One angle will not work for all $(\zeta, \eta)$ and $(z, w)$ and so a localization argument with many angles must be used.

\section{Main estimate}

In order to obtain convergence results, we will need an estimate on the real part of the exponent of our kernel. Various constants will emerge in the statement and proof of this estimate that depend on the compact set $K$ or $K^{\prime}$. We denote the dependency of a constant, such as $\delta$, on a set $A$ by writing $\delta_{A}$. We also use the following coordinates: the variable of integration $(\zeta, \eta)$ will always lie on $M$ and will be written as

$$
(\zeta, \eta)=\left(h\left(s, u^{\prime}, t\right)+i s, u^{\prime}+i t\right) \in M \quad \text { with } s \in \mathbf{R}, u^{\prime}, t \in \mathbf{R}^{n-1} .
$$

The point $(z, w)$ lies in $\mathbf{C} \times \mathbf{C}^{n-1}$ (not necessarily in $M$ ) and will be written as

$$
(z, w)=(h(y, u, v)+q+i y, u+i v) \quad \text { with } y, q \in \mathbf{R}, \quad u, v \in \mathbf{R}^{n-1} .
$$

Note that $q$ represents the vertical distance from the point $(z, w)$ to $M$. In particular $(z, w)$ belongs to $M$ if and only if $q=0$.

We now state the main estimate on the exponent of our kernel.

Lemma 1 (Main estimate). Suppose $K \subset K^{\prime}$ are given compact sets in $\mathbf{C}^{n}$. There exist constants $\Lambda_{K^{\prime}}, C_{K^{\prime}}, d_{K}, \delta_{K}, \delta_{K^{\prime}}, C_{K}>0$ and $p=p_{K^{\prime}}$ with $2<p<2 /\left(1-\delta_{K^{\prime}}\right)$ and an open cover $Q_{j}(j=1, \ldots, N)$ of $K$ and an open cover $Q_{k}^{\prime}\left(k=1, \ldots, N^{\prime}\right)$ of $K^{\prime}$ with diameters less than $d_{K} / 2$ and angles $\theta_{j, k}$ such that for all $\alpha \in C_{\theta_{j, k}}=\left\{r e^{i \theta_{j, k}} ; r>0\right\}$ and for all

$$
\begin{aligned}
(\zeta, \eta) & =\left(h\left(s, u^{\prime}, t\right)+i s, u^{\prime}+i t\right) \in Q_{k}^{\prime} \cap M \quad \text { and } \\
(z, w) & =(h(y, u, v)+q+i y, u+i v) \in Q_{j}
\end{aligned}
$$

(with $u, u^{\prime}, t, v \in \mathbf{R}^{n-1}$ and $s, y, q \in \mathbf{R}$ ) we have:

1) If $|s-y|<2 d_{K}$ for all $(z, w) \in Q_{j}$ and $(\zeta, \eta) \in Q_{k}^{\prime} \cap M$, then $\theta_{j, k}$ can be chosen with $\left|\theta_{j, k}\right|<\frac{\pi}{4}-\delta_{K}$ and

(1) $\operatorname{Re}\left(\alpha^{2}\left(\frac{\zeta-z}{\epsilon}\right)^{2}+\Lambda_{K^{\prime}} \alpha^{2}\left(\frac{w-\eta}{\epsilon}\right)^{2}-\alpha^{p}\right)$

$$
\leq-\delta_{K}\left(\left|\frac{s-y}{\epsilon}\right|^{2} r^{2}+\left|\frac{t-v}{\epsilon}\right|^{2} r^{2}+r^{p}\right)+C_{K^{\prime}}\left|\frac{u-u^{\prime}}{\epsilon}\right|^{2} r^{2}+C_{K}\left(\frac{q}{\epsilon}\right)^{2} r^{2} .
$$

2) If $|s-y| \geq d_{K}$ for all $(z, w) \in Q_{j}$ and $(\zeta, \eta) \in Q_{k}^{\prime} \cap M$, then $\theta_{j, k}$ can be chosen with $\left|\theta_{j, k}\right|<\frac{\pi}{4}-\delta_{K^{\prime}}$ and

$$
\begin{aligned}
& \operatorname{Re}\left(\alpha^{2}\left(\frac{\zeta-z}{\epsilon}\right)^{2}+\Lambda_{K^{\prime}} \alpha^{2}\left(\frac{w-\eta}{\epsilon}\right)^{2}-\alpha^{p}\right) \\
& \quad \leq-\delta_{K^{\prime}}\left(\left|\frac{s-y}{\epsilon}\right|^{2} r^{2}+\left|\frac{t-v}{\epsilon}\right|^{2} r^{2}+r^{p}\right)+C_{K^{\prime}}\left|\frac{u-u^{\prime}}{\epsilon}\right|^{2} r^{2} .
\end{aligned}
$$


Remark. Since the diameters of $Q_{j}$ and $Q_{k}^{\prime}$ are less than $d_{K} / 2$, each $Q_{j}$ and $Q_{k}^{\prime}$ falls under either case 1 or case 2 . In the second case, the constant, $\delta_{K^{\prime}}$, depends on $K^{\prime}$ (as opposed to $K$ for the constant in the first case). Since $K \subset K^{\prime}, \delta_{K^{\prime}}$ will generally be smaller than $\delta_{K}$. Also note that the estimate in the second case does not involve $q^{2}$.

Proof of the lemma. If $\widehat{W}$ is a complex number with $|\operatorname{Arg}(\widehat{W})| \geq 3 \delta_{1}>0$, there is an angle $\theta$ with $|\theta|<\frac{\pi}{4}-\delta_{1}$ such that $\operatorname{Re}\left\{e^{2 i \theta} \widehat{W}\right\}<0$. If we let $C_{\theta}=\left\{r e^{i \theta} ; r>0\right\}$, there is a $\delta_{2}>0$ such that $\operatorname{Re}\left\{\alpha^{2} \widehat{W}\right\} \leq-\delta_{2}|\widehat{W} \| \alpha|^{2}$ for all $\alpha \in C_{\theta}$. This observation will be used below to choose the angles $\theta_{j, k}$.

We first examine the term $(\zeta-z)^{2}$ that appears in the exponent of $E_{\epsilon}(\zeta, \eta, \alpha, z, w)$. We write

$$
\begin{aligned}
(\zeta, \eta) & =H\left(s, u^{\prime}, t\right)=\left(h\left(s, u^{\prime}, t\right)+i s, u^{\prime}+i t\right), \\
(z, w) & =H(y, u, v)+(q, 0)=(h(y, u, v)+i y+q, u+i v) .
\end{aligned}
$$

Note that $(\zeta, \eta)$ belongs to $M$; but $(z, w)$ belongs to $M$ if and only if $q=0$.

We wish to rotate $(\zeta-z)^{2}$ so that its real part is negative. For technical reasons that will be clear later, the rotation angle must be less than $\frac{\pi}{2}$. Thus, the troublesome case to handle is when $\zeta-z$ is a positive real number (which would then require a rotation angle greater than $\frac{\pi}{2}$ to make its real part negative). Therefore, we will handle separately the cases when $s-y=$ $\operatorname{Im}\{\zeta-z\}$ is small and not so small.

First, observe that there is a $\widetilde{\delta}_{K}>0$ such that

$$
\left|\operatorname{Arg}\left(h_{y}(y, u, v)+i\right)\right| \geq \widetilde{\delta}_{K} \quad \text { for all }(y, u, v) \in K .
$$

By choosing $d_{K}>0$ small enough and by shrinking $\widetilde{\delta}_{K}>0$, we can arrange

$$
\begin{aligned}
&\left|\operatorname{Arg}\left(\frac{h(s, u, v)-h(y, u, v)}{s-y}+i\right)\right| \geq \widetilde{\delta}_{K} \\
& \text { for all }(y, u, v) \in K, \text { and }|s-y| \leq 2 d_{K} .
\end{aligned}
$$

Choose a smooth function $\phi: \mathbf{R} \rightarrow[0,1]$ such that $\phi(s-y)=0$ for $|s-y| \leq d_{K} / 2$ and $\phi(s-y)=1$ for $|s-y| \geq d_{K}$.

After adding and subtracting terms, we obtain

$$
\begin{aligned}
\zeta-z= & h\left(s, u^{\prime}, t\right)-h(y, u, v)-q+i(s-y) \\
= & \left(\frac{h(s, u, v)-h(y, u, v)}{s-y}-\frac{\phi(s-y) q}{s-y}+i\right)(s-y) \\
& +\left(h\left(s, u^{\prime}, v\right)-h(s, u, v)\right)+\left(h\left(s, u^{\prime}, t\right)-h\left(s, u^{\prime}, v\right)\right) \\
& -(1-\phi(s-y)) q \\
= & W \cdot(s-y)+\left(h\left(s, u^{\prime}, v\right)-h(s, u, v)\right) \\
& +\left(h\left(s, u^{\prime}, t\right)-h\left(s, u^{\prime}, v\right)\right)-(1-\phi(s-y)) q
\end{aligned}
$$


where

$$
W=\frac{h(s, u, v)-h(y, u, v)}{s-y}-\frac{\phi(s-y) q}{s-y}+i .
$$

In view of (3) and the fact that $\phi(s-y)=0$ for $|s-y| \leq d_{K} / 2, \widetilde{\delta}_{K}$ can be shrunk, if necessary, so that $|\operatorname{Arg} W| \geq \widetilde{\delta}_{K}$ for $(y, u, v) \in K$ and $|s-y| \leq 2 d_{K}$. If $|s-y| \geq d_{K}$ with $H(s, u, v)$ belonging to the compact set $K^{\prime}$, then the inequality above holds with a constant $\widetilde{\delta}_{K^{\prime}}$ depending on $K^{\prime}$. We can then find an open cover (in $\mathbf{C}^{n}$ ) $Q_{1}, \ldots, Q_{N}$ of $K$ and an open cover $Q_{1}^{\prime}, \ldots, Q_{N^{\prime}}^{\prime}$ of $K^{\prime} \cap M$ with diameter smaller than $d_{K} / 2$ and angles $\theta_{j, k}$ and constants $\delta_{K}>0$ and $\delta_{K^{\prime}}>0$ such that:

Case 1. If $H(y, u, v)+(q, 0) \in Q_{j}$ and $H(s, u, v) \in Q_{k}^{\prime} \cap M$ with $|s-y| \leq 2 d_{K}$, then $\left|\theta_{j, k}\right| \leq \frac{\pi}{4}-\delta_{K}$ and

$$
\operatorname{Re}\left\{e^{2 i \theta_{j, k}} W^{2}\right\} \leq-\delta_{K}|W|^{2} \leq-\delta_{K} .
$$

Case 2. If $H(y, u, v)+(q, 0) \in Q_{j}$ and $H(s, u, v) \in Q_{k}^{\prime} \cap M$ with $|s-y| \geq d_{K}$, then $\left|\theta_{j, k}\right| \leq \frac{\pi}{4}-\delta_{K^{\prime}}$ and

$$
\operatorname{Re}\left\{e^{2 i \theta_{j, k}} W^{2}\right\} \leq-\delta_{K^{\prime}}|W|^{2} \leq-\delta_{K^{\prime}} .
$$

For shorthand in the next set of calculations, we let $\theta=\theta_{j, k}$. From (4), we obtain

$$
\begin{aligned}
& \operatorname{Re}\left(e^{2 i \theta}(\zeta-z)^{2}\right)=\operatorname{Re}\left(e^{2 i \theta} W^{2}(s-y)^{2}+e^{2 i \theta}\left(h\left(s, u^{\prime}, v\right)-h(s, u, v)\right)^{2}\right. \\
& \left.\quad+e^{2 i \theta}\left(h\left(s, u^{\prime}, t\right)-h\left(s, u^{\prime}, v\right)\right)^{2}+e^{2 i \theta}(1-\phi)^{2} q^{2}+\text { cross terms }\right) .
\end{aligned}
$$

For $(z, w)=H(y, u, v)+(q, 0) \in Q_{j}$ and $(\zeta, \eta)=H\left(s, u^{\prime}, t\right) \in Q_{k}^{\prime} \cap M$ with $|s-y| \leq 2 d_{K}$, we have

$$
\begin{aligned}
\left|h\left(s, u^{\prime}, v\right)-h(s, u, v)\right|^{2} & \leq C_{K^{\prime}}\left|u-u^{\prime}\right|^{2}, \\
\left|h\left(s, u^{\prime}, t\right)-h\left(s, u^{\prime}, v\right)\right|^{2} & \leq C_{K^{\prime}}|t-v|^{2},
\end{aligned}
$$

for some constant $C_{K^{\prime}}$ depending only on $K^{\prime}$. Therefore, in view of (5),

$$
\operatorname{Re}\left(e^{2 i \theta}(\zeta-z)^{2}\right) \leq-\frac{\delta_{K}}{2}|W|^{2}|s-y|^{2}+C_{K^{\prime}}\left(\left|u-u^{\prime}\right|^{2}+|t-v|^{2}\right)+C_{K} q^{2} .
$$

In this estimate we have handled the cross terms in the usual manner. For example, the cross term $W(s-y)(1-\phi) q$ is estimated as follows:

$|W(s-y)(1-\phi) q|=\sqrt{\frac{\delta_{K}}{4}} W|s-y| \cdot \sqrt{\frac{4}{\delta_{K}}}|q||1-\phi| \leq \frac{\delta_{K}}{4}|W|^{2}|s-y|^{2}+\frac{4}{\delta_{K}} q^{2}$.

The first term on the right can be absorbed by the $-\delta_{K}|W|^{2}$ term in (5). The second term on the right contributes to $C_{K} q^{2}$ appearing in (8). Other cross terms are handled similarly. 
In the case when $|s-y| \geq d_{K}$, we have $\phi(s-y)=1$, so the term in (7) involving $(1-\phi) q$ is zero. Therefore the preceding analysis can be repeated (using (6)) but without the term on the right involving $q$; that is, if $(z, w)=H(y, u, v)+(q, 0) \in Q_{j}$ and $(\zeta, \eta)=H\left(s, u^{\prime}, t\right) \in Q_{k}^{\prime} \cap M$ with $|s-y| \geq d_{K}$ then

$$
\operatorname{Re}\left(e^{2 i \theta}(\zeta-z)^{2}\right) \leq \frac{-\delta_{K^{\prime}}}{2}|W|^{2}|s-y|^{2}+C_{K^{\prime}}\left(\left|u-u^{\prime}\right|^{2}+|t-v|^{2}\right) .
$$

In (8), the angle $\theta$ is $\theta_{j k}$, satisfying $|\theta|=\left|\theta_{j, k}\right| \leq \frac{\pi}{4}-\delta_{K}$. In (9), the angle $\theta=\theta_{j k}$ satisfies $|\theta| \leq \frac{\pi}{4}-\delta_{K^{\prime}}$ (depending on the larger set $K^{\prime}$ ).

We now turn our attention to the terms involving $\eta-w$ in the exponent $E_{\epsilon}(\zeta, \eta, \alpha, z, w)$. If $|\theta| \leq \frac{\pi}{4}-\delta$, then

$$
\text { (10) } \begin{aligned}
\operatorname{Re} & \left(e^{2 i \theta}(\eta-w)^{2}\right) \\
& =\operatorname{Re}\left(e^{2 i \theta}\left(u^{\prime}-u+i(t-v)\right)^{2}\right) \\
& \leq-\cos \left(\frac{\pi}{2}-2 \delta\right)(t-v)^{2}+\left(u-u^{\prime}\right)^{2}+2 \sin \left(\frac{\pi}{2}-2 \delta\right)\left|u-u^{\prime}\right||t-v| \\
& \leq-(\delta)(t-v)^{2}+\left(u-u^{\prime}\right)^{2}+\frac{2 \sqrt{2}\left|u-u^{\prime}\right|}{\sqrt{\delta}} \frac{|t-v| \sqrt{\delta}}{\sqrt{2}} \\
& \left.\leq-\frac{\delta}{2}|t-v|^{2}+\left(1+\frac{2}{\delta}\right)\left|u-u^{\prime}\right|^{2} \quad \text { (using } 2|a||b| \leq a^{2}+b^{2}\right)
\end{aligned}
$$

for all $\eta$ and $w$. In the case $|s-y| \leq 2 d_{K}$ the constant $\delta=\delta_{K}$ depends on $K$, whereas in the case $|s-y| \geq d_{K}$ the constant $\delta=\delta_{K^{\prime}}$ depends on the larger set $K^{\prime}$.

Now choose a $p$ with $2<p<2 /(1-\delta)$, where $\delta$ is either $\delta_{K}$ or $\delta_{K^{\prime}}$. Then since $|\theta| \leq \frac{\pi}{4}-\delta$, we have $p|\theta|<\frac{\pi}{2}$; so there is a $\widetilde{\delta}>0$ such that

$$
\text { if } \alpha=r e^{i \theta} \text {, with } r \geq 0, \quad \text { then } \operatorname{Re} \alpha^{p} \geq \widetilde{\delta} r^{p} \text {. }
$$

Here, $\widetilde{\delta}$ depends either on $K$, in the case $|s-y| \leq 2 d_{K}$, or on $K^{\prime}$, in the case $|s-y| \geq d_{K}$.

In the case $|s-y| \leq 2 d_{K}$, we can combine the estimates given in (8) (noting that $|W| \geq 1$ ), (10), and (11), to obtain, with $\alpha=r e^{i \theta}$ :

$$
\begin{aligned}
\operatorname{Re}\left(\alpha^{2}\left(\frac{\zeta-z}{\epsilon}\right)^{2}+\Lambda \alpha^{2}\left(\frac{w-\eta}{\epsilon}\right)^{2}-\alpha^{p}\right) & \\
\leq- & \frac{\delta_{K}}{2}\left|\frac{s-y}{\epsilon}\right|^{2} r^{2}+C_{K^{\prime}}\left(\left|\frac{u-u^{\prime}}{\epsilon}\right|^{2}+\left|\frac{t-v}{\epsilon}\right|^{2}\right) r^{2} \\
& \quad+C_{K}\left(\frac{q}{\epsilon}\right)^{2} r^{2}-\frac{\delta_{K}}{2} \Lambda\left|\frac{t-v}{\epsilon}\right|^{2} r^{2}+\Lambda\left(1+\frac{2}{\delta_{K}}\right)\left|\frac{u-u^{\prime}}{\epsilon}\right|^{2} r^{2}-\widetilde{\delta}_{K} r^{p} .
\end{aligned}
$$

Choosing $\Lambda\left(=\Lambda_{K^{\prime}}\right)$ large enough so that $\Lambda \delta_{K} / 2>C_{K^{\prime}}+\delta_{K} / 2$ we obtain (1) after relabeling $\delta_{K}$ to be the smaller of $\delta_{K} / 2$ and $\widetilde{\delta}_{K}$ and relabeling as $C_{K^{\prime}}$ the quantity $C_{K^{\prime}}+\Lambda\left(1+2 / \delta_{K}\right)$. 
For the case when $|s-y| \geq d_{K}$, we use estimate (9) instead of (8) and the constant $\delta=\delta_{K^{\prime}}$ now depends on the larger set $K^{\prime}$. The only difference in the estimates above is that the right side no longer involves the term $q^{2}$ (compare (9) with (8)). Thus, (2) is obtained and this completes the proof of the main estimate given in the lemma.

\section{Approximation to the identity}

We restate the definition of our basic kernel:

$$
E_{\epsilon}(\zeta, \eta, \alpha, z, w)=\exp \left(\alpha^{2}\left(\frac{\zeta-z}{\epsilon}\right)^{2}+\alpha^{2} \Lambda_{K^{\prime}}\left(\frac{w-\eta}{\epsilon}\right)^{2}-\alpha^{p}\right) .
$$

Let $Q=Q_{j}$ and $Q^{\prime}=Q_{k}^{\prime}$ be the open sets and let $\theta=\theta_{j, k}$ be the angle provided by Lemma 1 . Suppose $f$ is continuous with compact support in $Q^{\prime} \cap M$. Define

$$
E_{\epsilon}(f)(z, w)=\frac{C}{\epsilon^{n}} \int_{(\zeta, \eta) \in M_{u}} \int_{\alpha \in C_{\theta}} f(\zeta, \eta) E_{\epsilon}(\zeta, \eta, \alpha, z, w) \alpha^{n} d \alpha d \zeta d \eta,
$$

where $C$ is a normalizing constant to be chosen later, $u=\operatorname{Re} w$ and

$$
M_{u}=\{(\zeta, u+i t) \in M\}=\{\operatorname{Re} \eta=u\} \cap M
$$

is a totally real $n$-dimensional submanifold of $M$ parameterized by

$$
(s, t) \in \mathbf{R} \times \mathbf{R}^{n-1} \mapsto(h(s, u, t)+i s, u+i t) .
$$

Here and below, $d \eta=d \eta_{1} \wedge \cdots \wedge d \eta_{n-1}$. The domain of integration in $E_{\epsilon}(f)(z, w)$ is $M_{u}$, which depends on $\operatorname{Re} w=u$. Since $p>2$ in the main estimate (Lemma 1), and since $f$ has compact support in $Q^{\prime} \cap M$, the expression $E_{\epsilon}(f)(z, w)$ is well-defined for $(z, w) \in Q$. This main estimate will also allow us to prove the following approximation result:

Lemma 2. Suppose $f$ is continuous with compact support in $Q^{\prime} \cap M$. Then

$$
E_{\epsilon}(f) \rightarrow f \quad \text { as } \epsilon \rightarrow 0
$$

uniformly on $Q \cap M$ (here, $Q=Q_{j}$ and $Q^{\prime}=Q_{k}^{\prime}$ are the open sets as in Lemma 1).

Proof. We analyze the exponent

$$
Z=\alpha^{2}\left(\frac{\zeta-z}{\epsilon}\right)^{2}+\alpha^{2} \Lambda_{K^{\prime}}\left(\frac{w-\eta}{\epsilon}\right)^{2}-\alpha^{p}
$$

in the case where

$$
(\zeta, \eta)=(h(s, u, t)+i s, u+i t) \in M_{u}
$$

(in particular, $u^{\prime}=u$ on $M_{u}$ ) and

$$
(z, w)=(h(y, u, v)+i y, u+i v)
$$


(i.e., $(z, w)$ belongs to $M$ and so $q=0)$. We obtain

$$
\begin{aligned}
\zeta-z & =h(s, u, t)-h(y, u, v)+i(s-y) \in \mathbf{C}, \\
\eta-w & =i(t-v) \in \mathbf{C}^{n-1} .
\end{aligned}
$$

With $\alpha=r e^{i \theta}$, the exponent of our kernel is

$$
\begin{aligned}
Z & =\alpha^{2}\left(\frac{\zeta-z}{\epsilon}\right)^{2}+\alpha^{2} \Lambda_{K^{\prime}}\left(\frac{w-\eta}{\epsilon}\right)^{2}-\alpha^{p} \\
& =e^{2 i \theta} r^{2}\left(\frac{h(s, u, t)-h(y, u, v)+i(s-y)}{\epsilon}\right)^{2}-\Lambda_{K^{\prime}}\left(\frac{t-v}{\epsilon}\right)^{2} e^{2 i \theta} r^{2}-e^{i p \theta} r^{p} .
\end{aligned}
$$

Now let

$$
\hat{s}=\frac{s-y}{\epsilon} \in \mathbf{R}, \quad \hat{t}=\frac{t-v}{\epsilon} \in \mathbf{R}^{n-1} .
$$

Estimates (1) and (2) with $u=u^{\prime}$ and $q=0$ essentially reduce to the same estimate except that the $\delta$-constant depends on $K$ in the first estimate and on $K^{\prime}$ in the second estimate. Since $K \subset K^{\prime}$, we will assume that $\delta_{K} \geq \delta_{K^{\prime}}$. In the hat-variables, these estimates (with $u=u^{\prime}$ and $q=0$ ) become

$$
\operatorname{Re} Z \leq-\delta_{K^{\prime}}\left(|\hat{s}|^{2} r^{2}+|\hat{t}|^{2} r^{2}+r^{p}\right) .
$$

The function

$$
e(\hat{s}, \hat{t}, r)=r^{n} \exp \left(-\delta_{K^{\prime}}\left(|\hat{s}|^{2} r^{2}+|\hat{t}|^{2} r^{2}+r^{p}\right)\right)
$$

is an integrable function on the set $\left\{(\hat{s}, \hat{t}, r) \in \mathbf{R} \times \mathbf{R}^{n-1} \times \mathbf{R}_{+} ; r \geq 0\right\}$ (to see this, integrate $\hat{s}$ and $\hat{t}$ and then integrate $r)$.

The Dominated Convergence Theorem now allows us to let $\epsilon \rightarrow 0$ in the integrand. The resulting exponent becomes

$$
Z=e^{2 i \theta} r^{2}\left(\left(\frac{\partial h(y, u, v)}{\partial y}+i\right) \hat{s}+\frac{\partial h}{\partial v}(y, u, v) \cdot \hat{t}\right)^{2}-\Lambda_{K^{\prime}} \hat{t}^{2} r^{2} e^{2 i \theta}-r^{p} e^{i p \theta} .
$$

In addition, $\zeta=(h(y+\epsilon \hat{s}, u, v+\epsilon \hat{t})+i(y+\epsilon \hat{s}))$ converges to $h(y, u, v)+i y=z$ uniformly for $(z, w) \in K$ as $\epsilon \rightarrow 0$. Likewise, $\alpha^{n} \epsilon^{-n} d \zeta \wedge d \eta \wedge d \alpha$ approaches $i^{n-1} r^{n} e^{i(n+1) \theta}\left(h_{y}(y, u, v)+i\right) d \hat{s} d \hat{t} d r$.

Now we rewrite the first part of the exponent involving $\hat{s}$ as

$$
\begin{aligned}
e^{2 i \theta} r^{2}\left(\left(\frac{\partial h(y, u, v)}{\partial y}+i\right)\right. & \left.\hat{s}+\frac{\partial h}{\partial v}(y, u, v) \cdot \hat{t}\right)^{2} \\
& =-r^{2}\left((-i) e^{i \theta} W \hat{s}+(-i) e^{i \theta} \frac{\partial h}{\partial v}(y, u, v) \cdot \hat{t}\right)^{2}
\end{aligned}
$$


where $W=\frac{\partial h(y, u, v)}{\partial y}+i \in \mathbf{C}$. We have

$$
\begin{aligned}
& \lim _{\epsilon \rightarrow 0} E_{\epsilon}(f)(z, w)=e^{i(n+1) \theta} C f(z, w) \\
& \times \int_{\hat{s}, \hat{t}} \int_{r \geq 0} \exp \left(-r^{2}\left(-i e^{i \theta} W \hat{s}-i e^{i \theta} \frac{\partial h}{\partial v}(y, u, v) \hat{t}\right)^{2}-\Lambda_{K^{\prime}} \hat{t}^{2} r^{2} e^{2 i \theta}-r^{p} e^{i p \theta}\right) \\
& \times r^{n} i^{n-1}\left(h_{y}(y, u, v)+i\right) d \hat{s} d \hat{t} d r .
\end{aligned}
$$

Using the limiting case as $s \rightarrow y$ in (5), we obtain $\operatorname{Re}\left(e^{2 i \theta}(W)^{2}\right)<0$. Therefore

$$
\operatorname{Re}\left(-i e^{i \theta} W \hat{s}\right)^{2}>0 .
$$

The idea now is to view the $\hat{s}$-integral in $E_{\epsilon}(f)$ as a contour integral over the contour $C$ given by

$$
\begin{aligned}
\hat{s} \mapsto z & =-i e^{i \theta} W \hat{s} \quad \text { for } \hat{s} \in \mathbf{R}, \\
\text { with } \quad d z & =-i e^{i \theta}\left(h_{y}(y, u, v)+i\right) d \hat{s} .
\end{aligned}
$$

The integral is $\int_{C} e^{-r^{2}(z+c)^{2}} d z$, where $c=-i e^{i \theta} h_{v}(y, u, v) \hat{t}$. In view of (12), $e^{-r^{2}(z+c)^{2}}$ is negatively exponentially decreasing on $C$. Cauchy's theorem implies that this contour integral is the same as $\int_{-\infty}^{\infty} e^{-r^{2}(s+c)^{2}} d s$. Therefore,

$$
\begin{aligned}
& \lim _{\epsilon \rightarrow 0} E_{\epsilon}(f)(z, w)=-i^{n-2} e^{i n \theta} C f(z, w) \\
& \times \int_{s, \hat{t}} \int_{r \geq 0} \exp \left(-r^{2}\left(s-i e^{i \theta} \frac{\partial h}{\partial v}(y, u, v) \hat{t}\right)^{2}-\Lambda_{K^{\prime}} \hat{t}^{2} r^{2} e^{2 i \theta}-r^{p} e^{i p \theta}\right) \\
& r^{n} d s d \hat{t} d r .
\end{aligned}
$$

Now we make a change of variables:

$$
\begin{aligned}
& \widetilde{s}=s+a \hat{t}, \quad \text { with } \quad a=(-i) e^{i \theta} \frac{\partial h}{\partial v}(y, u, v) \in \mathbf{C}^{n-1}, \\
& \widetilde{t}=\hat{t} \in \mathbf{R}^{n-1} .
\end{aligned}
$$

The Jacobian of the derivative of this change of variables is 1 . If $a$ were a real number, we could change variables in the usual manner and obtain (after dropping the tilde)

$$
\int_{s, \hat{t}} e^{-r^{2}(s+a \hat{t})^{2}-r^{2} \Lambda_{K^{\prime}} \hat{t}^{2} e^{2 i \theta}} d s d \hat{t}=\int_{s, t} e^{-r^{2} s^{2}-r^{2} \Lambda_{K^{\prime}} t^{2} e^{2 i \theta}} d s d t .
$$

Increasing $\Lambda_{K^{\prime}}$ if necessary, the left side is an analytic function of $a$ in a neighborhood of the ball $|a| \leq R$, where $R=\sup \left|h_{v}\right|$ over $K$. Therefore the preceding equality holds (by the identity theorem for analytic functions) for 
complex $a$ as well. We thus obtain

$$
\begin{aligned}
& \lim _{\epsilon \rightarrow 0} E_{\epsilon}(f)(z, w) \\
& \quad=-i^{n-2} e^{i n \theta} C f(z, w) \int_{s, t} \int_{r \geq 0} \exp \left(-r^{2} s^{2}-\Lambda_{K^{\prime}} t^{2} r^{2} e^{2 i \theta}-r^{p} e^{i p \theta}\right) r^{n} d s d t d r .
\end{aligned}
$$

We can now replace $e^{i \theta} t \in \mathbf{C}^{n-1}$ by $\tilde{t} \in \mathbf{R}^{n-1}$ and $e^{i(n-1) \theta} d t$ by $d \widetilde{t}$. This change of variables is justified, again, by Cauchy's Theorem and the fact that $|\theta|<\frac{\pi}{4}$. The result is (dropping the tilde)

$$
\begin{aligned}
& \lim _{\epsilon \rightarrow 0} E_{\epsilon}(f)(z, w) \\
& \quad=-i^{n-2} e^{i \theta} C f(z, w) \int_{s, t} \int_{r \geq 0} \exp \left(-r^{2} s^{2}-\Lambda_{K^{\prime}} t^{2} r^{2}-r^{p} e^{i p \theta}\right) r^{n} d s d t d r .
\end{aligned}
$$

We change variables by letting $\hat{t}=r t \in \mathbf{R}^{n-1}$ and $\hat{s}=r s \in \mathbf{R}$ (with $\left.r^{n} d s d t=d \hat{s} d \hat{t}\right)$. After integrating $\hat{s}$ and $\hat{t}$, the right side becomes

$$
C f(z, w) \cdot \text { Const }_{\Lambda_{K^{\prime}}} \int_{0}^{\infty} \exp \left(-r^{p} e^{i p \theta}\right) e^{i \theta} d r
$$

where Const $\Lambda_{K^{\prime}} \in \mathbf{C}$ is a constant depending only on $\Lambda_{K^{\prime}}$. We can view the preceding integral as an integral over the contour

$$
C_{\theta}=\left\{r \mapsto z=r e^{i \theta}\right\}, \quad \text { with } d z=e^{i \theta} d r .
$$

Since $|\theta|<\frac{\pi}{4}$, this contour lies in the right half-plane. Using the principal branch of $z^{p}$, the contour integral becomes

$$
C f(z, w) \cdot \text { Const }_{\Lambda_{K^{\prime}}} \int_{z \in C_{\theta}} e^{-z^{p}} d z .
$$

Since the integrand is analytic and rapidly decreasing at infinity (recall that $|p \theta|<\frac{\pi}{2}$ ), Cauchy's Theorem can be used to transform this integral into the following one over the positive real axis:

$$
C f(z, w) \cdot \text { Const }_{\Lambda_{K^{\prime}}} \int_{0}^{\infty} e^{-\hat{r}^{p}} d \hat{r} .
$$

This is now independent of $\theta$. Since the $\hat{r}$-integral converges, we may choose $C$ (as a normalizing constant) so that the expression above becomes $f(z, w)$. This completes the proof of Lemma 2.

\section{Fixing the slice $M_{u}$}

So far, the function $f$ is only required to be continuous (not CR). However, the domain of integration in $E_{\epsilon}(f)(z, w)$ is $M_{u}=\{\operatorname{Re} \eta=u\}$, which depends on $u=\operatorname{Re} w$. So despite the fact that the kernel is entire in $(z, w)$, the expression $E_{\epsilon}(f)(z, w)$ is not holomorphic in $w$. The next major step is to show that if $f$ is CR, then the domain can be fixed at a particular slice $M_{u_{0}}$ (independent of $w$ ). Then, the resulting integral will be holomorphic in both 
$z$ and $w$. Since $f$ is assumed to only have support on a small neighborhood of a fixed point (see Lemma 1), a localization argument with a partition of unity is needed. This adds some complicating facets since a partition of unity is not CR.

To get started with the next step, we first cover $M \cap K^{\prime}$ with an open cover $Q_{k}^{\prime}\left(k=1,2, \ldots, N^{\prime}\right)$, where each $Q_{k}^{\prime}$ satisfies the properties given in Lemma 1. Let $\phi_{k}$ be a partition of unity subordinate to this cover. Fix any $(z, w) \in K$ and let $Q_{j}$ be an open set that satisfies Lemma 1 and is of the form $Q_{j}=I \times J$, where $I \subset \mathbf{R}^{n-1}$ is an open set containing $\operatorname{Re} w$ and $J$ is an open set in $\mathbf{C} \times \mathbf{R}^{n-1}$ containing $(z, \mathrm{Im} \mathrm{w})$. Let $\theta_{j, k}$ be the angle given in Lemma 1 corresponding the open sets $Q_{k}^{\prime}$ in the coordinates $(\zeta, \eta)$ and $Q_{j}$ in the coordinates $(z, w)$. For the moment, the index $j$ will be fixed and $k$ will vary. Therefore, we will suppress the index $j$ and write $Q=Q_{j}$ and $\theta_{Q, k}=\theta_{j, k}$. For any $u_{0} \in I$, define

$$
F_{\epsilon}^{u_{0}, Q}(f)(z, w)=\sum_{k=1}^{N^{\prime}} \int_{(\zeta, \eta) \in M_{u_{0}}} \int_{\alpha \in C_{\theta_{Q}, k}}\left(\phi_{k} f\right)(\zeta, \eta) E_{\epsilon}(\zeta, \eta, \alpha, z, w) \alpha^{n} d \zeta d \eta d \alpha .
$$

From Lemma 2, $F_{\epsilon}^{u_{0}, Q}(f) \rightarrow f$ as $\epsilon \rightarrow 0$ uniformly on the set $Q \cap M_{u_{0}}$.

The next lemma contains the key step in fixing the domain of integration independently of Re $w$. In this lemma, the size of $K^{\prime} \cap M$ (the set on which $f$ is CR) will be determined based on the size of the original set $K$. Increasing the size of $K^{\prime}$ will add terms to the sum above (i.e., additional open sets, $Q_{k}^{\prime}$, and additional partition of unity functions $\phi_{k}$ may be required). However as long as $K^{\prime}$ is compact (which it will be), the sum will be finite.

Lemma 3. Suppose $K$ is a compact set in $\mathbf{C}^{n}$; then $K^{\prime} \subset \mathbf{C}^{n}$ can be chosen large enough (containing $K$ ) so that the following holds: suppose $f$ is $\mathrm{CR}$ on $K^{\prime} \cap M$. Let $Q=I \times J$ be the open set described above. There exists $\Delta_{K^{\prime}}>0$ such that if the diameter of $I$ is less than $\Delta_{K^{\prime}}$ and if $u_{0}, u_{1} \in I$, then $\left|\left(F_{\epsilon}^{u_{1}, Q}-F_{\epsilon}^{u_{0}, Q}\right)(f)(z, w)\right| \rightarrow 0$ as $\epsilon \rightarrow 0$ uniformly for $(z, w) \in Q=I \times J$.

Proof. Assume that $K^{\prime}$ is a closed ball of radius $R^{\prime}+1$ in $\mathbf{C}^{n}$. Since $f$ is CR on $K^{\prime} \cap M$, we can multiply $f$ by an appropriate cut-off function and assume that $f$ has compact support in $K^{\prime} \cap M$ and is $\bar{\partial}$-closed on the intersection of $M$ with the ball of radius $R^{\prime}$.

$M_{u_{0}}$ and $M_{u_{1}}$ are totally real $n$-dimensional slices of $M$ that naturally form the boundary of a real $n+1$-dimensional submanifold $\widetilde{M}_{0,1} \subset M$. In fact, just connect $u_{0}$ and $u_{1}$ by a real line segment and let $\widetilde{M}_{0,1}$ be the graph of $h$ over the $n+1$ real-dimensional strip spanned by this line segment and the $n$-dimensional subspace $\left\{\operatorname{Re} \eta=u_{0}\right\}$ of $\mathbf{R}^{2 n-1}$.

Since $E_{\epsilon}$ is holomorphic, Stokes' Theorem implies

$$
\left(F_{\epsilon}^{u_{1}, Q}-F_{\epsilon}^{u_{0}, Q}\right)(f)(z, w)=S_{1}+S_{2}
$$


with

$$
\begin{aligned}
& S_{1}=\sum_{k=1}^{N^{\prime}} \int_{(\zeta, \eta) \in \widetilde{M}_{01}} \int_{\alpha \in C_{\theta_{Q}, k}}\left(\bar{\partial} \phi_{k} f\right)(\zeta, \eta) E_{\epsilon}(\zeta, \eta, \alpha, z, w) \alpha^{n} d \zeta d \eta d \alpha \\
& S_{2}=\sum_{k=1}^{N^{\prime}} \int_{(\zeta, \eta) \in \widetilde{M}_{01}} \int_{\alpha \in C_{\theta_{Q, k}}}\left(\phi_{k} \bar{\partial} f\right)(\zeta, \eta) E_{\epsilon}(\zeta, \eta, \alpha, z, w) \alpha^{n} d \zeta d \eta d \alpha
\end{aligned}
$$

We first show that the sum in (14) converges to zero as $\epsilon \rightarrow 0$. The point $(\zeta, \eta)=\left(h\left(s, u^{\prime}, t\right)+i s, u^{\prime}+i t\right)$ must lie in the support of $\bar{\partial} f$, which lies outside the ball of radius $R^{\prime} \approx \operatorname{radius}\left(K^{\prime}\right)$. Also note that $u^{\prime}$ belongs to the line segment connecting $u_{0}$ and $u_{1}$, which in turn belongs to $K$ (or rather, the projection of $K$ onto the $u$-axis $)$. The point $(z, w)=(h(y, u, v)+q+i y, u+i v)$ lies in the smaller compact set $K$. We are at liberty to take $R^{\prime}$ as large as we please (relative to the diameter of $K$ ) in order to make the integrand in (14) converge to zero as $\epsilon \rightarrow 0$. Since $(z, w)$ and $u, u^{\prime}$ belong to $K$, we may choose $R^{\prime}$ large enough so that either

$$
|s-y| \geq \frac{R^{\prime}}{2} \quad \text { or } \quad|t-v| \geq \frac{R^{\prime}}{2}
$$

for all $(\zeta, \eta)$ belonging to the support of $\bar{\partial} f$.

We have two cases to consider on the exponent

$$
Z=\alpha^{2}\left(\frac{\zeta-z}{\epsilon}\right)^{2}+\alpha^{2} \Lambda_{K^{\prime}}\left(\frac{w-\eta}{\epsilon}\right)^{2}-\alpha^{p} .
$$

The first is $|s-y| \leq 2 d_{K}$, in which case we repeat the estimate (1):

$\operatorname{Re} Z \leq-\delta_{K}\left(\left|\frac{s-y}{\epsilon}\right|^{2} r^{2}+\left|\frac{t-v}{\epsilon}\right|^{2} r^{2}+r^{p}\right)+C_{K^{\prime}}\left|\frac{u-u^{\prime}}{\epsilon}\right|^{2} r^{2}+C_{K}\left(\frac{q}{\epsilon}\right)^{2} r^{2}$.

Now $u, u^{\prime}$ belong to $I$ and we will require the diameter of $I$ to be less than $\Delta_{K^{\prime}}$ (where $\Delta_{K^{\prime}}$ will be chosen below). In view of the inequality above and (15), we have

$$
\operatorname{Re} Z \leq \frac{-\delta_{K}\left(R^{\prime} / 2\right)^{2} r^{2}+C_{K^{\prime}} \Delta_{K^{\prime}}^{2} r^{2}+C_{K} q^{2} r^{2}}{\epsilon^{2}}
$$

Choose $R^{\prime}$ large enough that $-\delta_{K}\left(R^{\prime} / 2\right)^{2}+C_{K} q^{2}<-2$ for all $q$ in $K$. This choice of $R^{\prime}$ fixes the constant $C_{K^{\prime}}$. Now choose $\Delta_{K^{\prime}}$ small enough that $C_{K^{\prime}} \Delta_{K^{\prime}}^{2}<1$. Then the real part of exponent is at most $-r^{2} /\left(\epsilon^{2}\right)$.

In the case where $|s-y| \geq d_{K}$, (2) implies

$$
\operatorname{Re} Z \leq-\delta_{K^{\prime}}\left(\left|\frac{s-y}{\epsilon}\right|^{2} r^{2}+\left|\frac{t-v}{\epsilon}\right|^{2} r^{2}+r^{p}\right)+C_{K^{\prime}}\left|\frac{u-u^{\prime}}{\epsilon}\right|^{2} r^{2} .
$$

In view of $(15)$

$$
\operatorname{Re} Z \leq-\frac{\delta_{K^{\prime}} R^{\prime 2} r^{2}}{4 \epsilon^{2}}+\frac{C_{K^{\prime}} \Delta_{K^{\prime}}^{2} r^{2}}{\epsilon^{2}}
$$


By shrinking $\Delta_{K^{\prime}}$ we can arrange

$$
\operatorname{Re} Z \leq-\frac{\delta_{K^{\prime}} R^{2} r^{2}}{8 \epsilon^{2}}
$$

In either case $\left(|s-y| \geq d_{K}\right.$ or $\left.|s-y| \leq 2 d_{K}\right)$, the integrand of each term in (14) is dominated by $C e^{-r^{2} / \epsilon^{2}}$ or $C e^{-\delta_{K^{\prime}} R^{\prime 2} r^{2} /\left(8 \epsilon^{2}\right)}$ provided $u, u^{\prime} \in I$ and the diameter of $I$ is less than $\Delta_{K^{\prime}}$.

Since $\int_{0}^{\infty} e^{-r^{2} / \epsilon^{2}} d r \rightarrow 0$ and $\int_{0}^{\infty} e^{-\delta_{K^{\prime}}{R^{\prime}}^{2} r^{2} /\left(8 \epsilon^{2}\right)} d r \rightarrow 0$ as $\epsilon \rightarrow 0$, we conclude that the sum in (14) converges to zero.

Now we examine the sum $S_{1}$ in (13), which we restate as

$$
\sum_{k=1}^{N^{\prime}} \int_{(\zeta, \eta) \in \widetilde{M}_{01}} \int_{\alpha \in C_{\theta_{Q, k}}}\left(\bar{\partial} \phi_{k} f\right)(\zeta, \eta) E_{\epsilon}(\zeta, \eta, \alpha, z, w) \alpha^{n} d \zeta d \eta d \alpha .
$$

We have already determined the compact set $K^{\prime}$ (the size of which had to be chosen large enough to make the term in (14) converge to zero as $\epsilon \rightarrow 0$ ). Thus, all the constants in our integral kernels (i.e., $\Lambda_{K^{\prime}}$ and $p=p_{K^{\prime}}>2$ ) are now determined.

Note that the kernel $E_{\epsilon}(\zeta, \eta, \alpha, z, w)$ in the sum immediately above is the same for all $k$. The only terms that appear to vary with $k$ are the cutoff functions $\phi_{k}$ and the contours $C_{\theta_{Q, k}}$. The following lemma states that the integral of the kernel is independent of this contour.

Sublemma 1. Let $Q_{1}^{\prime}$ and $Q_{2}^{\prime}$ be intersecting open sets from our cover. Let $\theta_{1}$ and $\theta_{2}$ be any two angles that satisfy the requirements of Lemma 1 relative to $Q_{1}^{\prime}$ and $Q_{2}^{\prime}$ (in particular, $\left.\left|\theta_{1}\right|,\left|\theta_{2}\right|<\frac{\pi}{4}-\delta_{K^{\prime}}\right)$. Then

$$
\int_{\alpha \in C_{\theta_{1}}} E_{\epsilon}(\zeta, \eta, \alpha, z, w) \alpha^{n} d \alpha=\int_{\alpha \in C_{\theta_{2}}} E_{\epsilon}(\zeta, \eta, \alpha, z, w) \alpha^{n} d \alpha
$$

To prove this, recall that $C_{\theta}$ is a ray in the complex plane that makes an angle $\theta$ with the positive real axis. For $R>0$, let $A_{R, \theta_{1}, \theta_{2}}$ be the arc of a circle of radius $R$ that lies between $C_{\theta_{1}}$ and $C_{\theta_{2}}$ :

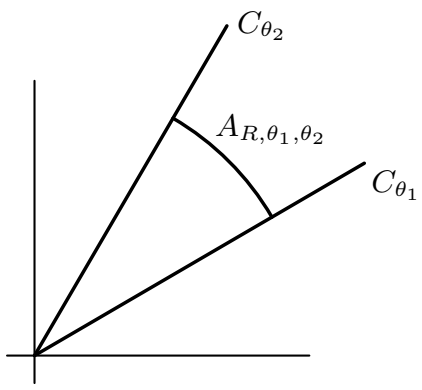


Since $E_{\epsilon}(\zeta, \eta, \alpha, z, w)$ is holomorphic in $\alpha$, Equation (16) will follow from Cauchy's Theorem provided we show that

$$
\sup _{\alpha \in A_{R, \theta_{1}, \theta_{2}}} R^{n+1}\left|E_{\epsilon}(\zeta, \eta, \alpha, z, w)\right| \rightarrow 0 \quad \text { as } R \rightarrow \infty .
$$

We restate the kernel:

$$
E_{\epsilon}(\zeta, \eta, \alpha, z, w)=\exp \left(\alpha^{2}\left(\frac{\zeta-z}{\epsilon}\right)^{2}+\alpha^{2} \Lambda_{K^{\prime}}\left(\frac{w-\eta}{\epsilon}\right)^{2}-\alpha^{p}\right) .
$$

Keep in mind that $\epsilon, \zeta, \eta, z$ and $w$ are fixed. Since $p>2$, the dominant term in the exponent is $\alpha^{p}$ as $|\alpha|=R \rightarrow \infty$. Let $\alpha=R e^{i \phi}$ be an arbitrary point on the arc $A_{R, \theta_{1}, \theta_{2}}$, so $\theta_{1} \leq \phi \leq \theta_{2}$. Since $|p \phi| \leq p\left|\max \left(\theta_{1}, \theta_{2}\right)\right|<\frac{\pi}{2}$ by the choice of $p$, there exists $\delta>0$ such that $-\operatorname{Re} \alpha^{p} \leq-\delta R^{p}$ for $\alpha \in A_{R, \theta_{1}, \theta_{2}}$. Equation (17) now follows since $R^{n+1} e^{-\delta R^{p}} \rightarrow 0$ as $R \rightarrow \infty$. This concludes the proof of the sublemma.

Using the fact that $\sum_{j} \phi_{j}=1$, the sum in (13) can be rewritten as

$$
\sum_{j=1}^{N^{\prime}} \sum_{k=1}^{N^{\prime}} \int_{(\zeta, \eta) \in \widetilde{M}_{01}} \int_{\alpha \in C_{\theta_{Q, k}}} \phi_{j}\left(\bar{\partial} \phi_{k} f\right)(\zeta, \eta) E_{\epsilon}(\zeta, \eta, \alpha, z, w) \alpha^{n} d \zeta d \eta d \alpha
$$

The support of $\phi_{j} \bar{\partial} \phi_{k}$ is contained in $Q_{j}^{\prime} \cap Q_{k}^{\prime} \cap M$. Using Equation (16), the integral over $C_{\theta_{Q, k}}$ can be replaced by the integral over $C_{\theta_{Q, j}}$. Therefore (18) becomes

$$
\sum_{j, k=1}^{N^{\prime}} \int_{(\zeta, \eta) \in \widetilde{M}_{01}} \int_{\alpha \in C_{\theta_{Q}, j}} \phi_{j}\left(\bar{\partial} \phi_{k} f\right)(\zeta, \eta) E_{\epsilon}(\zeta, \eta, \alpha, z, w) \alpha^{n} d \zeta d \eta d \alpha .
$$

Summing out $k$ we see that this term is zero because $\sum_{k} \bar{\partial} \phi_{k}=0$ on $M \cap K^{\prime}$. Thus the term in (13) is zero. Since the terms in (14) converge to zero as $\epsilon \rightarrow 0$, the proof of Lemma 3 is now complete.

Lemmas 2 and 3 imply:

Corollary 1. With the notation of Lemma 3, suppose $f$ is $\mathrm{CR}$ on $K^{\prime} \cap M$ and suppose that $Q=I \times J$, where the diameter of $I$ is less than $\Delta_{K^{\prime}}$. If $u_{0}$ belongs to $I$, then

$$
F_{\epsilon}^{u_{0}, Q}(f)(z, w) \rightarrow f \quad \text { as } \epsilon \rightarrow 0,
$$

uniformly for $(z, w) \in Q \cap M$.

\section{Globalization}

Now $F_{\epsilon}^{u_{0}, Q}(f)$ is holomorphic on $Q$ and $F_{\epsilon}^{u_{0}, Q}(f) \rightarrow f$ on $Q \cap M$. The set $Q$ is an appropriately small open set about an arbitrary point $(z, w)$ in $K$. Our next and final step is to piece together these locally defined holomorphic functions into a sequence of functions that are globally holomorphic on $K$ 
with the corresponding convergence to $f$ on $K \cap M$. This will require solving a $\bar{\partial}$ problem on $K$ with estimates.

To get started, we will assume $K$ is a ball (in particular, $K$ is a convex domain and so we can solve $\bar{\partial}$ on $K)$. We cover $K$ with open sets $Q_{j}$ $(j=1, \ldots, N)$ of the form $Q_{j}=I_{j} \times J_{j}$ where $I_{j}$ is an open set in $\mathbf{R}^{n-1}$ (with coordinates $u$ ) and $J_{j}$ is an open set in $\mathbf{R}^{n+1}=\mathbf{R} \times \mathbf{R} \times \mathbf{R}^{n-1}$ (with coordinates $\left.x \in \mathbf{R}, y \in \mathbf{R}, v \in \mathbf{R}^{n-1}\right)$. We assume that $Q_{j}$ is small enough to satisfy the requirements of Lemma 3 and that the diameter of each $I_{j}$ is smaller than $\Delta_{K^{\prime}}$ (from Lemma 3 ). Choose points $u_{j} \in I_{j}, j=1 \ldots N$. We let $\psi_{j}$ be a partition of unity for $K$ subordinate to the cover $Q_{j}$. We define

$$
\begin{aligned}
F_{\epsilon}(f)(z, w)= & \left(\sum_{j} \psi_{j} F_{\epsilon}^{u_{j}, Q_{j}}(f)(z, w)\right)-v_{\epsilon}(z, w) \\
= & \sum_{j, k} \psi_{j}(z, w) \int_{(\zeta, \eta) \in M_{u_{j}}} \int_{\alpha \in C_{\theta_{Q_{j}, k}}}\left(\phi_{k} f\right)(\zeta, \eta) \\
& \times E_{\epsilon}(\zeta, \eta, \alpha, z, w) \alpha^{n} d \zeta d \eta d \alpha-v_{\epsilon}(z, w),
\end{aligned}
$$

where $v_{\epsilon}$ will be chosen so that $F_{\epsilon}(f)$ is holomorphic in $K$. We will also show that $\left|v_{\epsilon}\right|$ converges to zero uniformly on $K$ as $\epsilon \rightarrow 0$. Then, Corollary 1 will imply that $F_{\epsilon}(f) \rightarrow f$ uniformly on $M \cap K$, as desired.

In order to arrange that $F_{\epsilon}(f)$ is holomorphic on $K$, we must require

$$
\begin{aligned}
\bar{\partial} v_{\epsilon}(z, w) & =\sum_{j} \bar{\partial}\left\{\psi_{j}\right\} F^{u_{j}, Q_{j}}(f)(z, w) \\
& =\sum_{l} \psi_{l} \sum_{j} \bar{\partial}\left\{\psi_{j}\right\} F_{\epsilon}^{u_{j}, Q_{j}}(f)(z, w),
\end{aligned}
$$

using $\sum_{l} \psi_{l}=1$ on $K$. If $\psi_{l} \bar{\partial}\left\{\psi_{j}\right\}$ is nonzero, $Q_{l}$ and $Q_{j}$ must overlap. We wish to change $F_{\epsilon}^{u_{j}, Q_{j}}$ to $F_{\epsilon}^{u_{l}, Q_{l}}$ in the sum above. Changing $Q_{j}$ to $Q_{l}$ involves changing the angle of the contour from $C_{\theta_{Q_{j}, k}}$ to $C_{\theta_{Q_{l}, k}}$ on the second line of (19), which Sublemma 1 allows us to do. Changing $u_{j}$ to $u_{l}$ is allowed by Lemma 3 but with a resulting error that tends to zero with $\epsilon$. Therefore, (20) becomes

$$
\bar{\partial} v_{\epsilon}(z, w)=\sum_{l} \psi_{l} \sum_{j} \bar{\partial}\left\{\psi_{j}\right\} F_{\epsilon}^{u_{l}, Q_{l}}(f)(z, w)+O(\epsilon),
$$

where $O(\epsilon)$ stands for terms that converge to zero uniformly on $K$ as $\epsilon \rightarrow 0$. Summing out $j$ and using the fact that $\sum_{j} \bar{\partial}\left\{\psi_{j}\right\}=0$, we conclude that $\bar{\partial} v_{\epsilon}=O(\epsilon)$ on $K$. Solving this $\bar{\partial}$ equation with sup-norm estimates on $K$, we can find a solution with $\left|v_{\epsilon}\right|=O(\epsilon)$. Now returning to (19) and using Corollary 1, we conclude that $F_{\epsilon}(f)$ is analytic on $K$ and converges uniformly to $f$ on $M \cap K$. Since $K$ is convex, it is also polynomially convex. 
Therefore, there is also a sequence of polynomials that converge uniformly on $K \cap M$ to $f$. This concludes the proof of our main theorem.

\section{References}

[BT] M.S. Baouendi and F. Trèves, A property of the functions and distributions annihilated by a locally integrable system of complex vector fields, Ann. of Math. (2), 113 (1981), 387-421, MR 0607899 (82f:35057), Zbl 0491.35036.

[B] A. Boggess, $C R$ extension for $L^{p} C R$ functions on a quadric submanifold of $\mathbf{C}^{n}$, Pacific J. Math., 201(1) (2001), 1-18, MR 1867889 (2002m:32051).

[Ch] E.M. Chirka, On the uniform approximation on totally real sets in $\mathbf{C}^{n}$, in 'Complex methods in approximation theory' (Almeria, 1995), 23-31, Monogr. Cienc. Tecnol., 2, Univ. Almeria, Almeria, 1997, 23-31, MR 1634170 (99k:32021), Zbl 0942.32014.

[DG] R. Dwilewicz and P.M. Gauthier, Global holomorphic approximations of CR functions on CR manifolds, Complex Variables Theory Appl., 4 (1985), 377-391, MR 0858919 (88b:32041), Zbl 0548.32011.

[HW] F.R. Harvey and R.O. Wells, Jr., Holomorphic approximation and hyperfunction theory on a $C^{1}$ totally real submanifold of a complex manifold, Math. Ann., 197 (1972), 287-318, MR 0310278 (46 \#9379), Zbl 0246.32019.

[N] J. Nunemacher, Approximation theory on totally real submanifolds, Math. Ann., 224 (1976), 129-141, MR 0422684 (54 \#10670), Zbl 0333.32015.

Received June 16, 2003. The second author was partially supported by Polish Committee for Scientific Research Grant KBN 2 P03A 04415.

Department of Mathematics

TEXAs A\&M University

College Station TX 77843-3368

E-mail address: boggess@math.tamu.edu

DEPARTMENT OF MATHEMATICS AND STATISTICS

UNIVERSITY OF MisSOURI

Rolla MO 65409

E-mail address: romand@umr.edu 